\title{
Rapid Molecular Detection of Tuberculosis and Rifampin
}

\section{Resistance}

Catharina C. Boehme, M.D., Pamela Nabeta, M.D., Doris Hillemann, Ph.D., Mark P. Nicol, Ph.D., Shubhada Shenai, Ph.D., Fiorella Krapp, M.D., Jenny Allen, B.Tech., Rasim Tahirli, M.D., Robert Blakemore, B.S., Roxana Rustomjee, M.D., Ph.D., Ana Milovic, M.S., Martin Jones, Ph.D., Sean M. O'Brien, Ph.D., David H. Persing, M.D., Ph.D., Sabine RueschGerdes, M.D., Eduardo Gotuzzo, M.D., Camilla Rodrigues, M.D., David Alland, M.D., and Mark D. Perkins, M.D.

Foundation for Innovative New Diagnostics, Geneva (C.C.B., P.N., M.D.P.); Forschungszentrum Borstel, Borstel, Germany (D.H., S.R.-G.); the Department of Clinical Laboratory Sciences, University of Cape Town, and National Health Laboratory Service, Cape Town (M.P.N., A.M.), and the Unit for Clinical and Biomedical TB Research, South African Medical Research Council, Durban (J.A., R.R.) - all in South Africa; P.D. Hinduja National Hospital and Medical Research Centre (Hinduja), Mumbai, India (S.S., C.R.); Instituto de Medicina Tropical Alexander von Humboldt, Universidad Peruana Cayetano Heredia, Lima, Peru (F.K., E.G.); Special Treatment Institution, Baku, Azerbaijan (R.T.); the Division of Infectious Diseases, New Jersey Medical School, University of Medicine and Dentistry of New Jersey, Newark (R.B., D.A.); Cepheid, Sunnyvale, CA (M.J., D.H.P.); and the Department of Biostatistics and Bioinformatics, Duke University Medical Center, Durham, NC (S.M.O.).

\section{Abstract}

Background-Global control of tuberculosis is hampered by slow, insensitive diagnostic methods, particularly for the detection of drug-resistant forms and in patients with human immunodeficiency virus infection. Early detection is essential to reduce the death rate and interrupt transmission, but the complexity and infrastructure needs of sensitive methods limit their accessibility and effect.

\begin{abstract}
Methods-We assessed the performance of Xpert MTB/RIF, an automated molecular test for Mycobacterium tuberculosis (MTB) and resistance to rifampin (RIF), with fully integrated sample processing in 1730 patients with suspected drug-sensitive or multidrug-resistant pulmonary tuberculosis. Eligible patients in Peru, Azerbaijan, South Africa, and India provided three sputum specimens each. Two specimens were processed with $\mathrm{N}$-acetyl-L-cysteine and sodium hydroxide before microscopy, solid and liquid culture, and the MTB/RIF test, and one specimen was used for direct testing with microscopy and the MTB/RIF test.
\end{abstract}

\begin{abstract}
Results-Among culture-positive patients, a single, direct MTB/RIF test identified 551 of 561 patients with smear-positive tuberculosis $(98.2 \%)$ and 124 of 171 with smear-negative tuberculosis (72.5\%). The test was specific in 604 of 609 patients without tuberculosis $(99.2 \%)$. Among patients with smear-negative, culture-positive tuberculosis, the addition of a second MTB/ RIF test increased sensitivity by 12.6 percentage points and a third by 5.1 percentage points, to a total of $90.2 \%$. As compared with phenotypic drug-susceptibility testing, MTB/RIF testing correctly identified 200 of 205 patients (97.6\%) with rifampin-resistant bacteria and 504 of 514
\end{abstract}

Copyright (C) 2010 Massachusetts Medical Society.

Address reprint requests to Dr. Boehme at the Foundation for Innovative New Diagnostics, Ave. de Budé 16, 1202 Geneva, Switzerland, or at catharina.boehme@finddiagnostics.org.. 
(98.1\%) with rifampin-sensitive bacteria. Sequencing resolved all but two cases in favor of the MTB/RIF assay.

Conclusions-The MTB/RIF test provided sensitive detection of tuberculosis and rifampin resistance directly from untreated sputum in less than 2 hours with minimal hands-on time. (Funded by the Foundation for Innovative New Diagnostics.)

ONLY A SMALL FRACTION OF THE ESTIMATED 500,000 patients who have multidrug-resistant tuberculosis and 1.37 million patients who have coinfection with tuberculosis and the human immunodeficiency virus (HIV) worldwide each year have access to sufficiently sensitive case detection or drug-susceptibility testing.1 Diagnostic delay, aggravated by the disproportionate frequency of smear-negative disease in HIV-associated tuberculosis, is common.2-5 The failure to quickly recognize and treat affected patients leads to increased mortality, secondary resistance (including extensively drug-resistant tuberculosis), and ongoing transmission.6,7 The complexity of mycobacterial culture and current nucleic acid-amplification technologies for the detection of tuberculosis and multidrug-resistant tuberculosis8 and the need for the associated infrastructure restrict the use of such tests to reference laboratories.

To respond to the urgent need for simple and rapid diagnostic tools at the point of treatment in high-burden countries, ${ }^{9}$ a fully automated molecular test for tuberculosis case detection and drug-resistance testing was developed through collaboration in a public-private partnership. Xpert MTB/RIF, an automated molecular test for Mycobacterium tuberculosis (MTB) and resistance to rifampin (RIF), uses heminested real-time polymerase-chainreaction (PCR) assay to amplify an MTB-specific sequence of the $r p o B$ gene, which is probed with molecular beacons for mutations within the rifampin-resistance determining region.10,11 Testing is carried out on the MTB/RIF test platform (GeneXpert, Cepheid), which integrates sample processing and PCR in a disposable plastic cartridge containing all reagents required for bacterial lysis, nucleic acid extraction, amplification, and amplicon detection. ${ }^{12}$ The only manual step is the addition of a bactericidal buffer to sputum before transferring a defined volume to the cartridge. The MTB/RIF cartridge is then inserted into the GeneXpert device, which provides results within 2 hours.

In accordance with recommendations on design and conduct of diagnostic accuracy assessments, ${ }^{13}$ we undertook a multicenter, prospective evaluation of the MTB/RIF test to determine its sensitivity and specificity in the intended target population as compared with the best available reference standard.

\section{METHODS}

\section{STUDY POPULATION}

From July 2008 through March 2009, we conducted this study at five trial sites in Lima, Peru; Baku, Azerbaijan; Cape Town and Durban, South Africa; and Mumbai, India. We enrolled consecutive adults with symptoms suggestive of pulmonary tuberculosis or multidrug-resistant tuberculosis who were able to provide three sputum samples of at least $1.5 \mathrm{ml}$. Patients in the group at risk for pulmonary tuberculosis were eligible only if they had not received a tuberculosis medication within the past 60 days, whereas the group at risk for multidrug-resistant disease included patients who had undergone previous treatment, those with nonconverting pulmonary tuberculosis who were receiving therapy, and symptomatic contacts of patients with known multidrug-resistant disease. All patients were enrolled from populations that were selected for diversity in the prevalence of tuberculosis, HIV coinfection, and multidrug resistance. (For details regarding the sites, see the Supplementary Appendix, available with the full text of this article at NEJM.org.) 
The study protocol was reviewed and approved by eight institutional review boards or technical committees at the ministerial level. The study was conducted in accordance with the protocol (available at NEJM.org). Written informed consent was obtained from all patients. Study participation did not alter the standard of care.

\section{STUDY DESIGN AND OVERSIGHT}

This study was designed and supervised by the sponsor, the Foundation for Innovative New Diagnostics (FIND). Additional development support was provided by the National Institutes of Health, Cepheid, and the Bill and Melinda Gates Foundation, none of which were involved in the design or conduct of the study. Data were collected by investigators at each study site, and statistical analyses were performed by a statistician who was not involved in data collection. FIND authors wrote the first draft of the manuscript. All authors vouch for the accuracy and completeness of the data reported.

\section{LABORATORY METHODS}

Patients meeting the clinical eligibility criteria were asked to provide three sputum specimens over a 2-day period (two spot samples and one obtained in the morning) (Fig. 1). In a random fashion, two of the three samples were processed with $N$-acetyl-L-cysteine and sodium hydroxide (NALC-NaOH), 14 followed by centrifugation, and then were resuspended in $1.5 \mathrm{ml}$ of phosphate buffer and subjected to microscopy with Ziehl-Neelsen staining, and cultivation on solid medium (egg-based Löwenstein-Jensen15 or 7H11,16 with the latter medium used only in Durban) and liquid medium (BACTECMGIT [mycobacteria growth indicator tube] 960 culture; BD Microbiology Systems), and the MTB/RIF test. The third sputum sample was tested directly by Ziehl-Neelsen microscopy and the MTB/RIF test without NALC-NaOH decontamination.

The first positive culture from each specimen underwent confirmation of M. tuberculosis species by MPT64 antigen detection (Capilia TB, Tauns Laboratories) 17 and indirect drugsusceptibility testing with the proportion method on Löwenstein-Jensen medium (for sites in Lima, Durban, and Baku) or MGIT SIRE18 (for sites in Cape Town and Mumbai). For three sites, conventional nucleic acid-amplification testing was carried out on DNA that was extracted from the NALC-NaOH centrifugation pellet of the first sputum sample with the use of Cobas Amplicor MTB (Roche) (in Cape Town and Mumbai) or ProbeTec ET MTB Complex Direct Detection Assay (BD) (in Baku), according to the manufacturer's instructions. At three sites, drug-resistant genotyping was carried out by line-probe assay with the use of the Geno-type MTBDRplus assay (Hain Lifescience) performed from culture isolates (in Baku) or from the NALC-NaOH pellet of the second sputum sample (in Cape Town and Durban), according to the manufacturer's instructions, except that smear-negative specimens were also tested.

All participating laboratories were quality-assured reference laboratories. Study laboratories for four sites were located within $5 \mathrm{~km}$ of the enrollment clinic and tested samples within 2 days after collection. Sputum samples from Baku were shipped to the German National Reference Laboratory in Borstel for testing 1 to 5 days after collection.

Repeat tuberculosis analyses (smear, culture, MTB/RIF test, radiography, and clinical workup) were performed in patients who had smear- and culture-negative samples if the MTB/RIF test or other nucleic acid-amplification test was positive or if the patient was selected by the central database as a random control for follow-up. The final diagnosis for patients undergoing repeat analyses was established on the basis of conventional laboratory results and clinical information by clinical review committees composed of three local tuberculosis clinicians. HIV results were obtained by review of clinical records and were 
available for only a subgroup of patients. Bias was minimized through blinding, since technicians performing molecular and reference tests were not aware of the results of other tests. The interpretation of data from MTB/RIF tests was software-based and independent of the user. Clinical teams and review committees did not have access to nucleic acidamplification test results. All study coordinators received lists of patients for follow-up but not the reasons for follow-up.

\section{CATEGORIES FOR ANALYSIS}

Patients were divided into four categories for analysis: those with smear- and culturepositive pulmonary tuberculosis; those with smear-negative, culture-positive pulmonary tuberculosis; those with no bacteriologic evidence of tuberculosis who had improvement without treatment (no tuberculosis); and those who were smear- and culture-negative for pulmonary tuberculosis who nonetheless were treated for tuberculosis on the basis of clinical and radiologic findings (clinical tuberculosis). A smear-positive case was defined as at least two smears of scanty grade (1 to 10 acid-fast bacilli per 100 fields) or one or more smears of $1+$ or more (10 to 99 bacilli per 100 fields). A culture-positive case was defined as positive results on at least one of four culture vials. Because a clear final diagnosis was required, patients with an indeterminate diagnosis were excluded from the main analysis if there was a negative culture result while the patient was receiving tuberculosis treatment (for patients with suspected multidrug resistance), contamination of at least three of four cultures, growth of nontuberculous mycobacteria only, indeterminate phenotypic rifampin susceptibility, a negative culture with a positive sputum smear, or suspected crosscontamination of cultures (i.e., only one of four cultures had positive results after $>28$ days to growth in MGIT or $<20$ colonies in Löwenstein-Jensen medium) or if the patient died or was lost to follow-up.

\section{MTB/RIF TEST}

The MTB/RIF test was performed as described previously ${ }^{19,20}$ (Fig. 2). Two laboratory technicians were trained as operators and passed proficiency testing after four runs per person. Sample re-agent was added in a 2:1 ratio to untreated sputum and in a 3:1 ratio to decontaminated sputum pellets. The additional sample reagent in pellets was necessary to meet the volume requirements for the assay sample. The closed sputum container was manually agitated twice during a 15-minute period at room temperature before $2 \mathrm{ml}$ of the inactivated material was transferred to the test cartridge (equivalent to $0.7 \mathrm{ml}$ of untreated sputum or $0.5 \mathrm{ml}$ of decontaminated pellet). Cartridges were inserted into the test platform, which was located in the microscopy room or another general-purpose laboratory space. The electronic results were sent directly from the MTB/RIF test system to the central database.

\section{SEQUENCING}

Bidirectional sequencing was performed on the 81-bp rроB core region of culture isolates in all rifampin-resistant and discordant strains with forward

(CGTGGAGGCGATCACACCGCAGAC) and reverse (AGCTCCAGCCCGGCACGCTCACGT) primers with the use of the BigDye Terminator Cycle Sequencing kit, according to the manufacturer's recommendations, in a 3130xl Genetic Analyzer (Applied Biosystems). Traces were analyzed with ABI sequence-analysis software, version 5.2.0.

\section{STATISTICAL ANALYSIS}

Sensitivity and specificity for the MTB/RIF test were estimated for a single direct test, a single test on a pelleted sample, the combination of two tests (one direct and one pelleted), and the combination of three tests (one direct and two pelleted). Combinations were 
classified as positive if at least one of the component test results was positive. The indeterminate rate was the number of tests classified as "invalid," "error," or "no result" divided by the total number performed. When results were indeterminate and sufficient sample remained, the assay was repeated once, and the second result was used for analysis. For analyzing the single direct test and the combination of three tests, Wilson's binomial method was used to calculate $95 \%$ confidence intervals. ${ }^{21}$ For all other intrapatient MTB/ RIF results, and for comparisons across subgroups and testing methods, generalized estimating equations were used for calculating confidence intervals to account for withinpatient clustering. 22

\section{RESULTS}

\section{PATIENTS}

Of the 1462 patients (4386 samples) included in the analysis, $567(38.8 \%)$ had smear- and culture-positive tuberculosis; 174 (11.9\%) had smear-negative, culture-positive tuberculosis; $105(7.2 \%)$ had clinically defined tuberculosis; and 616 (42.1\%) had no clinical evidence of tuberculosis (Table 1). Of patients with culture-positive samples, 207 of 741 (27.9\%) were found to have multidrug resistance on conventional drug-susceptibility testing. A total of 113 patients were not eligible for testing because of an inadequate number of sputum samples (in 103 patients) or an inadequate volume of sputum samples (in 10). A total of 268 patients were excluded from the analysis for a variety of reasons, including 115 who had culture-negative samples but were receiving tuberculosis treatment at enrollment because of suspected multidrug resistance (Fig. 1).

\section{SENSITIVITY AND SPECIFICITY}

Case Detection-Among patients with culture-positive tuberculosis, the overall sensitivity of the MTB/RIF test was $97.6 \%$. The sensitivity was $99.8 \%$ for smear- and culture-positive cases and $90.2 \%$ for smear-negative, culture-positive cases, with no significant variation in overall sensitivity across sites $(P=0.24$ by chi-square test $)$ (Table 2$)$. Testing of multiple specimens per patient had a modest effect over the yield of a single assay performed directly on sputum. The sensitivity of a single direct MTB/RIF test for culture-confirmed tuberculosis was $92.2 \%$ and rose to $96.0 \%$ with the additional testing of a pelleted sample. For the detection of smear-negative, culture-positive tuberculosis, the sensitivity of the assay was $72.5 \%$ for one test, $85.1 \%$ for two tests, and $90.2 \%$ for three tests. A single, direct MTB/RIF test identified a greater proportion of culture-positive patients than did a single Löwenstein-Jensen culture (Table 1 in the Supplementary Appendix). Among HIV-positive patients with pulmonary tuberculosis, the sensitivity of the MTB/RIF test was 93.9\%, as compared with $98.4 \%$ in HIV-negative patients $(\mathrm{P}=0.02)$. There was no significant difference in sensitivity between tests on untreated sputum and those on decontaminated pellet $(\mathrm{P}=0.16)$.

The estimated specificity was $99.2 \%$ for a single direct MTB/RIF test, $98.6 \%$ for two MTB/ RIF tests, and $98.1 \%$ for three MTB/RIF tests. At sites performing alternative nucleic acidamplification testing, the sensitivity of the MTB/RIF test performed directly on sputum was higher than that of Amplicor (94.6\% vs. 86.8\%, P<0.01) and similar to that of ProbeTec ( $83.7 \%$ vs. $83.9 \%, \mathrm{P}=0.96)$ performed on extracted DNA from sputum pellets. The specificity of the MTB/RIF test did not differ significantly from that of Amplicor or Probetec (Table 2 in the Supplementary Appendix).

Detection of Multidrug Resistance-Table 3 shows the sensitivity and specificity of the MTB/RIF test for the detection of rifampin and multidrug resistance (resistance to both rifampin and isoniazid). For 15 of 718 patients for whom results on the MTB/RIF test were 
discrepant on phenotypic testing, sequencing confirmed resistance-associated $r p o B$ mutations in nine strains that were identified as rifampin-sensitive on drug-susceptibility testing, determined the presence of a wild-type allele in one strain deemed rifampin-resistant on drug-susceptibility testing, and identified 3 patients with mixed infection containing wild-type and mutant strains in the same culture. Taking sequencing results into account, the MTB/RIF test correctly detected rifampin resistance in 209 of 211 patients $(99.1 \%$ sensitivity) and in all 506 patients with rifampin susceptibility (100\% specificity).

The $r p o B$ mutations found in this study were representative of the global situation: 16 different mutations were identified, but a limited number, notably in codons 516, 526 and 531, accounted for almost all resistant strains.

Using the South African samples, we compared the performance of the direct Genotype MTBDRplus assay with that of the MTB/RIF test. In smear-positive sputum samples, the MTBDRplus assay showed a sensitivity equivalent to that of the MTB/RIF test. However, in samples from smear-negative, culture-positive patients, for which the MTBDRplus assay is not indicated, the MTBDRplus assay provided a false negative result in 37 of 67 samples $(55.2 \%)$.

In a subgroup of 115 patients with culture-negative tuberculosis who had suspected multidrug resistance and were receiving tuberculosis treatment (and who were excluded from the main analysis), 51 had positive results on the MTB/RIF test, and rifampin resistance was detected in 8 . We observed that all 8 patients were later started on second-line therapy for treatment failure by physicians who were unaware of the results on MTB/RIF testing. In comparison, none of 8 randomly selected patients from the same cohort with positive results on MTB/RIF testing that did not detect rifampin resistance were given second-line tuberculosis treatment. Although the manufacturer currently recommends that the MTB/RIF test be used for patients with suspected tuberculosis who have not received treatment, our data provide a first indication that the test also detects multidrug-resistant tuberculosis in patients who are receiving therapy, even after culture conversion.

\section{INDETERMINATE RATE}

The MTB/RIF test was indeterminate in 192 of 5190 tests performed (3.7\%), a rate that was lower than the overall culture-contamination rate (5.5\%) in 381 of 6920 MGIT and Löwenstein-Jensen cultures $(\mathrm{P}<0.001)$. Allowing for one repeat test, the indeterminate rate dropped to $1.2 \%$ (63 of 5190 tests). Valid results were obtained in 129 of 139 repeat tests (92.8\%). No patient had indeterminate results on all samples tested. A total of 20 of 2072 samples $(1.0 \%)$ with positive results had an indeterminate result for rifampin resistance. These indeterminate rifampin results all occurred in smear-negative, culture-positive sputum samples with a very late cycle threshold (35 to 37 cycles) in the MTB/RIF test. A software change allowing the assay to analyze results for up to 40 cycles would have eliminated 19 of the 20 indeterminate results without affecting the specificity of the assay.

\section{DISCUSSION}

In our study, an assay that was designed for point-of-treatment use in low-income countries accurately detected pulmonary tuberculosis and screened for rifampin resistance. This assay identified more than $97 \%$ of all patients with culture-confirmed tuberculosis who met the inclusion criteria, including more than $90 \%$ of patients with smear-negative disease.

Performance both for case detection and discrimination of rifampin resistance was similar across diverse sites, suggesting that the findings are likely to be widely applicable. In view of the low sensitivity of smear microscopy for the diagnosis of tuberculosis in patients with HIV infection, the increased sensitivity of the MTB/RIF test — notably, among patients 
with smear-negative tuberculosis - at the two South African sites with 60 to $80 \%$ prevalence of HIV infection is encouraging.

There are several reasons why the findings of this study might not translate widely into improved care for patients with tuberculosis. First, only reference facilities were used in the study, and it is not certain that our findings would be replicated in microscopy centers, health posts, and other point-of-treatment settings where temperature and electricity supply will be more variable and training issues will be more relevant. However, qualitative questionnaires that were completed during the study suggested that users considered 2 to 3 days a sufficient duration of training for technicians without previous molecular experience (as compared with 2 weeks for Ziehl-Neelsen microscopy). The relative simplicity of the MTB/RIF test, plus its hands-on time of under 15 minutes and its unambiguous readout, is advantageous, whereas the need for annual calibration was identified as a challenge for implementation at peripheral laboratories, especially in rural areas. Large-scale projects to show the feasibility and effect of MTB/RIF testing at such sites are under way.

Second, to achieve great simplicity of use, the MTB/RIF test uses sophisticated technology, which is costly to manufacture. Although FIND has negotiated concessionary pricing for public-sector programs in low-income countries and is working to further lower the costs of testing, the costs of instruments and tests will still be considerably higher than those for microscopy, which is all that is currently available in peripheral health care settings in many countries. However, MTB/RIF testing could be less costly than implementation of culture and drug-susceptibility testing.

Globally, ineffective tuberculosis detection and the rise of multidrug resistance and extensively drug-resistant tuberculosis have led to calls for dramatic expansion of culture capability and drug-susceptibility testing in countries in which the disease is endemic. ${ }^{23}$ Unfortunately, the infrastructure and trained personnel required for such testing are not available except in a limited number of reference centers, and results of testing are often not available for at least 4 months, which dramatically reduces its clinical utility. ${ }^{24,25}$ The complexity of standard nucleic acid-amplification tests prevents the expansion of this method. The MTB/RIF test automates DNA extraction, amplification, and detection inside a test cartridge that is never reopened, with little chance of amplicon contamination. Specimen processing is simplified to a single nonprecise step that both liquefies and inactivates sputum, which results in a reduction in viable tubercle bacilli of 6 to 8 logs and eliminates the necessity for a biosafety cabinet. Data from a recent study confirm that the MTB/RIF assay generates no infectious aerosols. ${ }^{26}$ These features of simplicity and safety of use could allow for cost-effective and highly sensitive detection of tuberculosis and drug resistance outside reference centers, which would increase access to testing and decrease delays in diagnosis, without the need to build large numbers of laboratories equipped for advanced biosafety.

\section{Supplementary Material}

Refer to Web version on PubMed Central for supplementary material.

\section{Acknowledgments}

Supported by FIND (for clinical evaluation and assay development) and grants from the National Institutes of Health (AI52523), Cepheid, and the Bill and Melinda Gates Foundation (for assay development).

Disclosure forms provided by the authors are available with the full text of this article at NEJM.org.

We thank the clinical and laboratory teams at the partner sites for their efforts in the implementation, conduct, and timely completion of the study; Justine de Grandpré and Dr. Ranald Sutherland for their continuous technical 
support and project management during assay development; and FIND logistics officer Nora Champouillon and data manager Pierrick Gonnet for facilitating the project.

\section{References}

1. Global tuberculosis control - epidemiology, strategy, financing: WHO report. World Health Organization; Geneva: 2009. (WHO/HTM/TB/2009.411.)

2. Perkins MD, Cunningham J. Facing the crisis: improving the diagnosis of tuberculosis in the HIV era. J Infect Dis 2007;196(Suppl 1):S15-S27. [PubMed: 17624822]

3. Getahun H, Harrington M, O'Brien R, Nunn P. Diagnosis of smear-negative pulmonary tuberculosis in people with HIV infection or AIDS in resource-constrained settings: informing urgent policy changes. Lancet 2007;369:2042-9. [PubMed: 17574096]

4. Havlir DV, Getahun H, Sanne I, Nunn P. Opportunities and challenges for HIV care in overlapping HIV and TB epidemics. JAMA 2008;300:423-30. [PubMed: 18647985]

5. Uys PW, Warren RM, van Helden PD. A threshold value for the time delay to TB diagnosis. PLoS One 2007;2(1):e757. [PubMed: 17712405]

6. Farmer P, Bayona J, Becerra M, et al. The dilemma of MDR-TB in the global era. Int J Tuberc Lung Dis 1998;2:869-76. [PubMed: 9848606]

7. Van Rie A, Enarson D. XDR tuberculosis: an indicator of public-health negligence. Lancet 2006;368:1554-6. [PubMed: 17084741]

8. Palomino JC. Molecular detection, identification and drug resistance detection in Mycobacterium tuberculosis. FEMS Immunol Med Microbiol 2009;56:103-11. [PubMed: 19416361]

9. Urdea M, Penny LA, Olmsted SS, et al. Requirements for high impact diagnostics in the developing world. Nature 2006;444(Suppl 1):73-9. [PubMed: 17159896]

10. El-Hajj HH, Marras SA, Tyagi S, Kramer FR, Alland D. Detection of rifampin resistance in Mycobacterium tuberculosis in a single tube with molecular beacons. J Clin Microbiol 2001;39:4131-7. [PubMed: 11682541]

11. Piatek AS, Tyagi S, Pol AC, et al. Molecular beacon sequence analysis for detecting drug resistance in Mycobacterium tuberculosis. Nat Biotechnol 1998;16:359-63. [PubMed: 9555727]

12. Raja S, Ching J, Xi L, et al. Technology for automated, rapid, and quantitative PCR or reverse transcription-PCR clinical testing. Clin Chem 2005;51:882-90. [PubMed: 15746302]

13. Bossuyt PM, Reitsma JB, Bruns DE, et al. Towards complete and accurate reporting of studies of diagnostic accuracy: the STARD initiative: Standards for Reporting of Diagnostic Accuracy. Clin Chem 2003;49:1-6. [PubMed: 12507953]

14. Kent, PT.; Kubica, GP., editors. Public health mycobacteriology: a guide for the Level III laboratory. Centers for Disease Control; Atlanta: 1985.

15. Laboratory services in tuberculosis control. World Health Organization; Geneva: 1998. (WHO/TB/ 98.258.)

16. Cohn ML, Waggoner RF, McClatchy JK. The 7H11 medium for the cultivation of mycobacteria. Am Rev Respir Dis 1968;98:295-6. [PubMed: 4299186]

17. Hillemann D, Rüsch-Gerdes S, Richter E. Application of the Capilia TB assay for culture confirmation of Mycobacterium tuberculosis complex isolates. Int J Tuberc Lung Dis 2005;9:1409-11. [PubMed: 16466066]

18. Siddiqi, SH.; Ruesch-Gerdes, S. MGIT procedure manual for BACTEC MGIT 960 TB System. Beckton, Dickinson; Franklin Lakes, NJ: Jul. 2006

19. Helb D, Jones M, Story E, et al. Rapid detection of Mycobacterium tuberculosis and rifampin resistance by use of on-demand, near-patient technology. J Clin Microbiol 2010;48:229-37. [PubMed: 19864480]

20. Blakemore R, Story E, Helb D, et al. Evaluation of the analytical performance of the Xpert MTB/ RIF Assay. J Clin Microbiol 2010;48:2495-501. [PubMed: 20504986]

21. Agresti A, Coull B. Approximate is better than "exact" for interval estimation of binomial proportions. Am Stat 1998;52:119-26.

22. Zeger SL, Liang KY. Longitudinal data analysis for discrete and continuous outcomes. Biometrics 1986;42:121-30. [PubMed: 3719049] 
23. Stop TB Partnership: the global plan to stop TB 2006-2015. World Health Organization; Geneva: 2006.

24. Yagui M, Perales MT, Asencios L, et al. Timely diagnosis of MDR-TB under program conditions: is rapid drug susceptibility testing sufficient? Int J Tuberc Lung Dis 2006;10:838-43. [PubMed: 16898366]

25. Urbanczik R, Rieder HL. Scaling up tuberculosis culture services: a precautionary note. Int J Tuberc Lung Dis 2009;13:799-800. [PubMed: 19555526]

26. Banada PP, Sivasubramani SK, Blakemore R, et al. Containment of bioaerosol infection risk by the Xpert MTB/ RIF assay and its applicability to point-of-care settings. J Clin Microbiol. August $18 ; 2010$ (Epub ahead of print). 


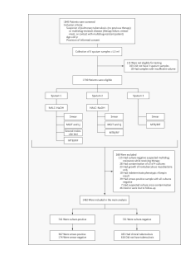

Figure 1. Enrollment and Outcomes

Patients were enrolled at centers that have diverse populations with a high prevalence of tuberculosis. In Lima, Peru, patients with suspected tuberculosis were enrolled at 30 primary care clinics with a high rate of tuberculosis case notification, a rate of coinfection with the human immunodeficiency virus (HIV) of less than $3 \%$, and a low rate of multidrug resistance. In Cape Town and Durban, South Africa, patients were enrolled at primary care tuberculosis clinics located within informal settlements with a high incidence of tuberculosis and an estimated rate of HIV coinfection of $70 \%$ and a rate of multidrug resistance of $4 \%$. In Mumbai, India, patients with complicated tuberculosis and a rate of multidrug resistance as high as $50 \%$ were enrolled at a tertiary care center. In Baku, Azerbaijan, prisoners were enrolled on arrival at a tuberculosis screening and treatment facility, which reports a high rate of multidrug resistance $(25 \%)$ among patients with tuberculosis and a rate of HIV coinfection of approximately 6\%. LJ denotes Löwenstein-Jensen, MGIT mycobacteria growth indicator tube, and NALC-NaOH N-acetyl-L-cysteine and sodium hydroxide. 


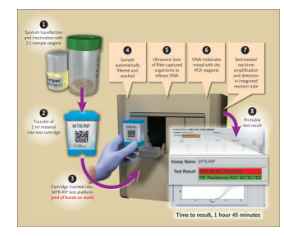

Figure 2. Assay Procedure for the MTB/RIF Test

Two volumes of sample treatment reagent are added to each volume of sputum. The mixture is shaken, incubated at room temperature for 15 minutes, and shaken again. Next, a sample of 2 to $3 \mathrm{ml}$ is transferred to the test cartridge, which is then loaded into the instrument. All subsequent steps occur automatically. The user is provided with a printable test result, such as "MTB detected; RIF resistance not detected." PCR denotes polymerase chain reaction. 


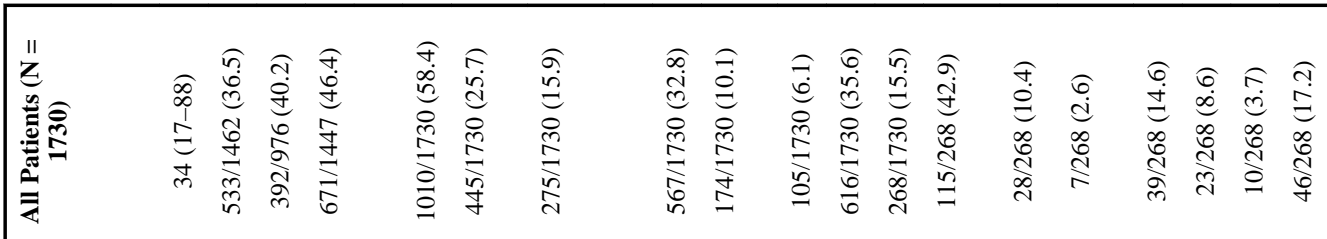

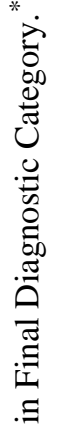

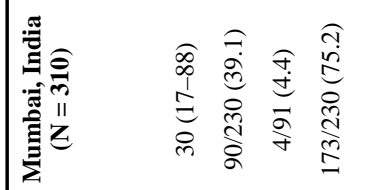

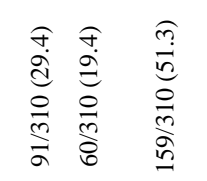

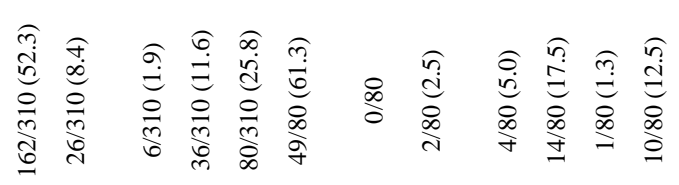

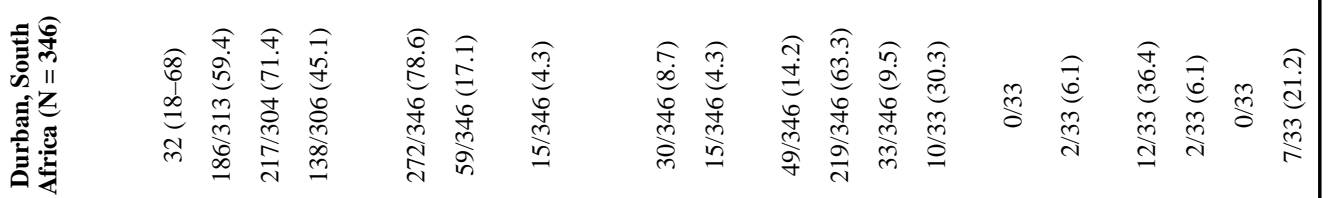
$\stackrel{\overbrace{}}{\Xi}$

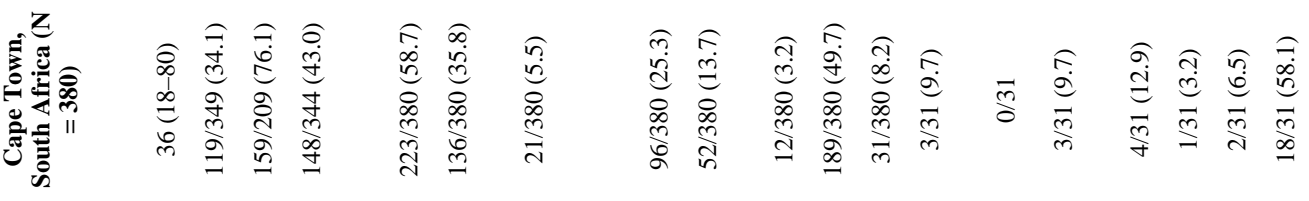

ส

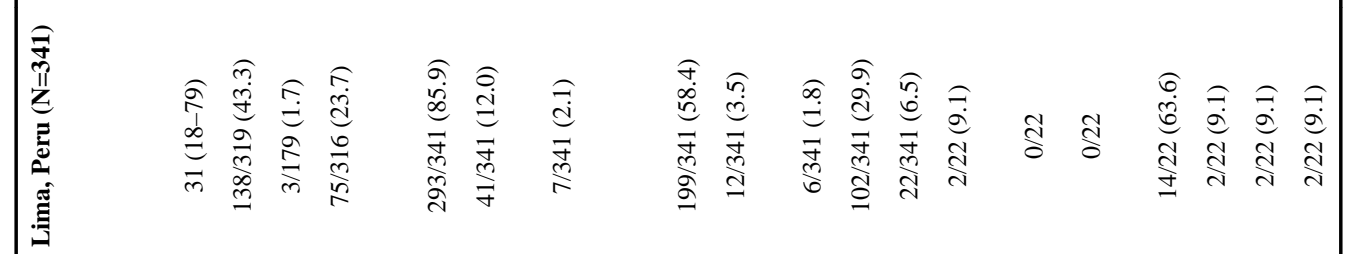

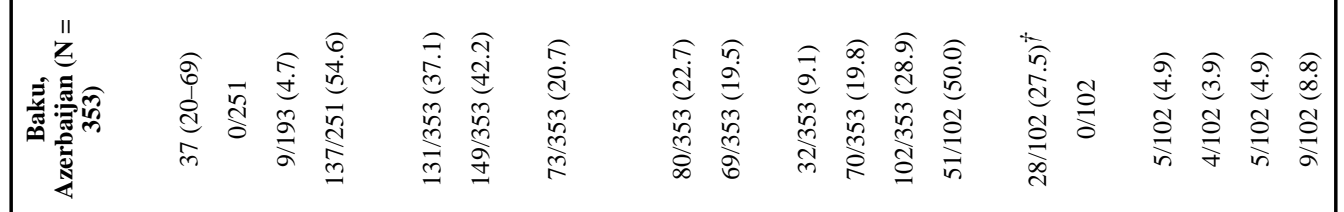

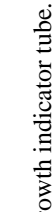

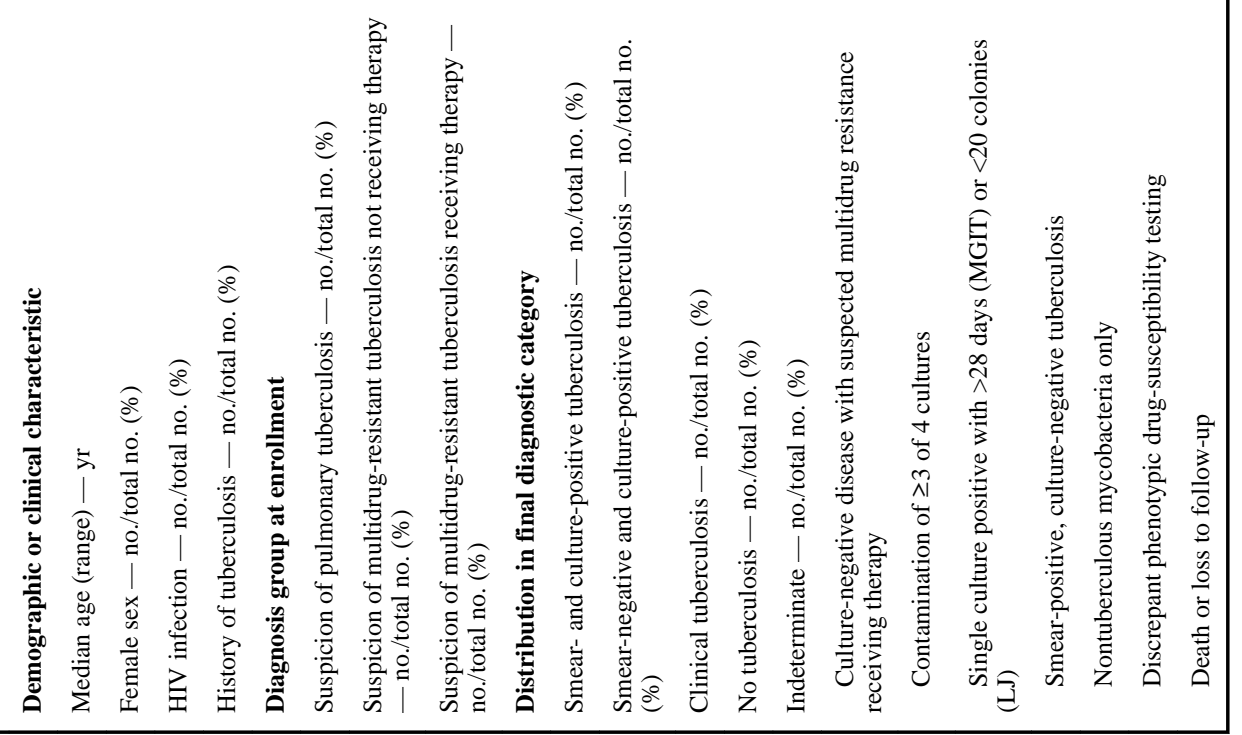


Table 2

Overall Sensitivity and Specificity of the MTB/RIF Test, According to the Number of Tests per Patient, as Compared with Three Smears and Four Cultures. ${ }^{*}$

\begin{tabular}{|c|c|c|c|c|}
\hline $\begin{array}{l}\text { Site and No. of } \\
\text { Tests }\end{array}$ & \multicolumn{2}{|r|}{ Sensitivity } & Smear-Negative and Culture-Positive & $\begin{array}{c}\text { Specificity } \\
\text { No Tuberculosi }\end{array}$ \\
\hline \multicolumn{5}{|l|}{ Site } \\
\hline \multicolumn{5}{|l|}{ Lima, Peru } \\
\hline $\begin{array}{l}\text { Correct - no./ } \\
\text { total no. }(\%)\end{array}$ & 209/211 (99.1) & 199/199 (100) & $10 / 12(83.3)$ & $102 / 102(100)$ \\
\hline $95 \% \mathrm{CI}$ & $96.6-99.7$ & $98.1-100.0$ & $55.2-95.3$ & $96.4-100.0$ \\
\hline \multicolumn{5}{|l|}{ Baku, Azerbaijan } \\
\hline $\begin{array}{l}\text { Correct - no./ } \\
\text { total no. }(\%)\end{array}$ & $144 / 149(96.6)$ & $80 / 80(100.0)$ & $64 / 69(92.8)$ & $68 / 70(97.1)$ \\
\hline $95 \% \mathrm{CI}$ & $92.4-98.6$ & $95.4-100.0$ & $84.1-96.9$ & $90.2-99.2$ \\
\hline \multicolumn{5}{|l|}{$\begin{array}{l}\text { Cape Town, South } \\
\text { Africa }\end{array}$} \\
\hline $\begin{array}{l}\text { Correct - no./ } \\
\text { total no. }(\%)\end{array}$ & $142 / 148(95.9)$ & $95 / 96(99.0)$ & $47 / 52(90.4)$ & $186 / 189(98.4)$ \\
\hline $95 \% \mathrm{CI}$ & $91.4-98.1$ & $94.3-99.8$ & $79.4-95.8$ & $95.4-99.5$ \\
\hline \multicolumn{5}{|l|}{ Durban, South Africa } \\
\hline $\begin{array}{l}\text { Correct }- \text { no./ } \\
\text { total no. }(\%)\end{array}$ & $43 / 45(95.6)$ & $30 / 30(100.0)$ & $13 / 15(86.7)$ & $213 / 219(97.3)$ \\
\hline $95 \% \mathrm{CI}$ & $85.2-98.8$ & $88.6-100.0$ & $62.1-96.3$ & $94.2-98.7$ \\
\hline \multicolumn{5}{|l|}{ Mumbai, India } \\
\hline $\begin{array}{l}\text { Correct - no./ } \\
\text { total no. }(\%)\end{array}$ & 185/188 (98.4) & $162 / 162(100.0)$ & $23 / 26(88.5)$ & $35 / 36(97.2)$ \\
\hline $95 \% \mathrm{CI}$ & $95.4-99.5$ & $99.7-100.0$ & $71.0-96.0$ & $85.8-99.5$ \\
\hline \multicolumn{5}{|l|}{$\begin{array}{l}\text { No. of MTB/RIF } \\
\text { tests }\end{array}$} \\
\hline \multicolumn{5}{|l|}{$\begin{array}{l}3 \text { Samples ( } 2 \text { pellet } \\
\text { and } 1 \text { direct) }\end{array}$} \\
\hline $\begin{array}{l}\text { Correct - no./ } \\
\text { total no. }(\%)\end{array}$ & 723/741 (97.6) & $566 / 567(99.8)$ & $157 / 174(90.2)$ & $604 / 616(98.1)$ \\
\hline $95 \% \mathrm{CI}$ & $96.2-98.5$ & $99.0-100.0$ & $84.9-93.8$ & $96.6-98.9$ \\
\hline \multicolumn{5}{|l|}{$\begin{array}{l}2 \text { Samples (1 pellet } \\
\text { and } 1 \text { direct) }\end{array}$} \\
\hline $\begin{array}{l}\text { Correct - no./ } \\
\text { total no. }(\%)^{\dagger}\end{array}$ & $1423 / 1482(96.0)$ & $1127 / 1134(99.4)$ & $296 / 348(85.1)$ & $1215 / 1232(98.6)$ \\
\hline $95 \% \mathrm{CI}$ & $94.6-97.1$ & $98.6-99.7$ & $79.7-89.2$ & $97.5-99.2$ \\
\hline \multicolumn{5}{|l|}{1 Sample (direct) } \\
\hline $\begin{array}{l}\text { Correct }- \text { no./ } \\
\text { total no. }(\%)\end{array}$ & $675 / 732(92.2)$ & $551 / 561(98.2)$ & $124 / 171(72.5)$ & $604 / 609$ (99.2) \\
\hline $95 \% \mathrm{CI}$ & $90.0-93.9$ & $96.8-99.0$ & $65.4-78.7$ & $98.1-99.6$ \\
\hline
\end{tabular}

Site-specific performance is shown for three MTB/RIF test results per patient (two pellet samples plus one direct sample). The sensitivity of the test did not differ significantly between patients who were suspected of having pulmonary tuberculosis and those suspected of having multidrugresistant tuberculosis $(\mathrm{P}=0.96)$. (For details, see Table 3 in the Supplementary Appendix.) Of 105 patients with culture-negative samples who were treated for tuberculosis on the basis of clinical symptoms, $29.3 \%$ had positive results on the MTB/RIF test (data not shown), but no further analysis was done during this study. 
${ }^{\dagger}$ The denominator for patients with two tests includes two observations per patient. The first observation is a combination of the first sputum sample (pellet) and third sputum sample (direct). The second observation is a combination of the second sputum sample (pellet) and the third sputum sample (direct). The calculation of the confidence interval (CI) accounts for within-patient correlation and the use of the third sputum sample two times. 


\section{Table 3}

Sensitivity and Specificity of the MTB/RIF Test for the Detection of Rifampin and Multidrug Resistance, as Compared with Phenotypic Drug-Susceptibility Testing Alone and in Combination with Sequencing of Discrepant Cases, According to Site. ${ }^{*}$

\begin{tabular}{|c|c|c|c|c|}
\hline \multirow[t]{2}{*}{ Site and Total } & \multicolumn{2}{|c|}{ Phenotypic Drug-Susceptibility Testing ${ }^{\dagger}$} & \multicolumn{2}{|c|}{$\begin{array}{l}\text { Phenotypic Drug-Susceptibility Testing and } \\
\text { Discrepant Resolution by Sequencing }{ }^{\dagger}\end{array}$} \\
\hline & $\begin{array}{c}\text { Sensitivity for } \\
\text { Rifampin Resistance }\end{array}$ & $\begin{array}{c}\text { Specificity for } \\
\text { Rifampin Resistance }\end{array}$ & $\begin{array}{c}\text { Sensitivity for } \\
\text { Rifampin Resistance }\end{array}$ & $\begin{array}{c}\text { Specificity for } \\
\text { Rifampin Resistance }\end{array}$ \\
\hline Lima, Peru — no./total no. (\%) & $16 / 16(100.0)$ & $190 / 193(98.4)$ & 19/19 (100.0) & $190 / 190(100.0)$ \\
\hline $\begin{array}{l}\text { Baku, Azerbaijan - no./total no. } \\
(\%)\end{array}$ & $47 / 49(95.9)$ & 90/94 (95.7) & $51 / 52(98.1)$ & $90 / 90(100.0)$ \\
\hline $\begin{array}{l}\text { Cape Town, South Africa - no./ } \\
\text { total no. }(\%)\end{array}$ & $15 / 16(93.8)$ & $126 / 126(100.0)$ & $15 / 15(100.0)$ & $126 / 126(100.0)$ \\
\hline $\begin{array}{l}\text { Durban, South Africa - no./total } \\
\text { no. }(\%)\end{array}$ & $3 / 3(100.0)$ & $38 / 38(100.0)$ & $3 / 3(100.0)$ & $38 / 38(100.0)$ \\
\hline Mumbai, India — no./total no. (\%) & $119 / 121(98.3)$ & $61 / 64(95.3)$ & $121 / 122(99.2)$ & $62 / 62(100.0)$ \\
\hline \multicolumn{5}{|l|}{ Total for rifampin resistance } \\
\hline Correct - no./total no. $(\%)$ & $200 / 205(97.6)$ & $505 / 515(98.1)$ & 209/211 (99.1) & $506 / 506(100.0)$ \\
\hline $95 \% \mathrm{CI}-\%$ & $94.4-99.0$ & $96.5-98.9$ & $96.6-99.7$ & $99.2-100.0$ \\
\hline \multicolumn{5}{|l|}{ Total for multidrug resistance } \\
\hline Correct - no. /total no. $(\%)$ & $195 / 200(97.5)$ & & $197 / 199(99.0)$ & \\
\hline $95 \% \mathrm{CI}-\%$ & $94.3-98.9$ & & $96.4-99.7$ & \\
\hline
\end{tabular}

